\title{
Listening for the Cosmic Hum of Black Holes
}

\section{A new analysis technique would allow the gravitational-wave "background" from distant black hole mergers to be detected in days instead of years.}

\section{by Neil Cornish*}

$\mathrm{T}$ he recent detection of gravitational waves from outer space has ushered in a new era of astronomy. To date, the teams behind these detections-the Laser Interferometer Gravitational-Wave Observatory (LIGO) and Virgo collaborations-have observed waves from a handful of black hole mergers [1-5] and one binary neutron star merger [6]. But these detected events are just the tip of the iceberg. Estimates suggest that a pair of stellarmass black holes, like the ones LIGO and Virgo observed, merge somewhere in the Universe every few minutes. For neutron star binaries, mergers occur every 15 seconds or so. Yet only a few of these mergers are sufficiently close by to produce "gold-plated" detections. A new paper explains how best to combine the signals from the multitude of less prominent merger events to reveal information about the Universe's entire black hole binary population [7]. The proposed technique, from Rory Smith and Eric Thrane of Monash University in Australia, will allow us to detect the background "rumble" of distant mergers far sooner than we would using traditional methods.

LIGO and Virgo detect gravitational waves by looking for minute changes in the distance between mirrors on either end of kilometer-scale laser interferometers. A passing gravitational wave effectively wiggles the mirrors back and forth, producing an oscillating signal that lasts a fraction of a second for black hole mergers and up to a few minutes for neutron star mergers. The US-based LIGO is comprised of two such interferometers separated by 3000 km, while Virgo has one interferometer in Italy.

The LIGO and Virgo teams consider a signal "significant" if, upon analysis, it has a greater than $99.99 \%$ probability of being of astrophysical origin. The five black hole mergers and the neutron star merger reported to date all fall into this category. By comparing the gravitational-wave signals from these events to predictions based on Einstein's theory of gravity, the researchers inferred the masses, spins, and

* Department of Physics, Montana State University, Bozeman, MT 59717, USA

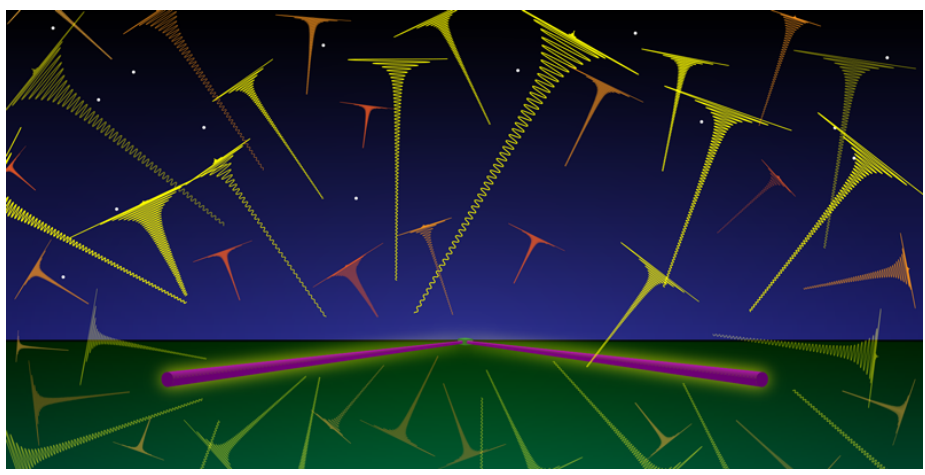

Figure 1: The majority of black hole mergers are too far away to create a significant signal in gravitational-wave detectors. The background from weaker signals, however, provides information about the entire black hole population. Smith and Thrane [7] have proposed a new analysis technique that would allow researchers to assess this gravitational-wave background much more rapidly. (APS/Alan Stonebraker)

sky location of the sources. The detections have also been used to infer the merger rate of black holes and neutron stars throughout the Universe.

At their current sensitivities, the LIGO and Virgo detectors can only detect a tiny fraction of the mergers in the Universe with high significance. The rest of the mergers contribute to a large number of subthreshold events-some barely distinguishable from noise in the detectors, others almost at the level of a real event. For example, the LIGO detectors picked up a promising event (LVT151012) in October of 2015. But a subsequent analysis determined that there was only an $87 \%$ probability of this event being from a black hole merger [2] - too low a number to claim detection.

To fully understand black mergers, many have to be observed, so it's a shame to discard the potentially valuable information from LVT151012 and other low-significance events $[8,9]$. Luckily, it's possible to learn something from the host of weaker signals that collectively form an unresolved background (Fig. 1). Think of listening to frogs croaking in a swamp: We can pick up clear songs from the nearest frogs, but we can also hear an indistinct hum from the thousands of frogs that are farther away. The volume of 
this background hum provides a measure of the frog population. Similarly, the amplitude of the unresolved background in LIGO and Virgo's detectors can tell us about distant black hole mergers that occurred when the Universe was much younger.

The traditional way to distinguish this gravitational-wave background signal from noise is to compare the outputs of two or more detectors with a so-called cross-correlation analysis. Noise is uncorrelated between detectors, so its contribution will average to zero in a cross-correlation, while any gravitational-wave signal should survive. Predictions suggest that, using the cross-correlation technique, the LIGO and Virgo detectors should be sensitive enough to detect the gravitational-wave background within several years (see 31 March 2016 Focus story) [10].

But as Smith and Thrane point out in their paper, crosscorrelation is only optimal for randomly shaped, and potentially overlapping, signals that produce a relatively constant background. Instead, the signals from black hole mergers tend to be nonoverlapping in detectors, and their shape (or time profile) has a specific form that's dictated by the properties of the black holes (Fig. 1). The researchers therefore suggest an alternative approach that should be thousands of times more sensitive than the traditional one. Their proposal entails first breaking up the raw data from each of LIGO and Virgo's three interferometers into short segments of a few seconds. The next step is to jointly analyze the same time segment from all interferometers to determine the probability that a black hole merger (as opposed to just noise) occurred. Critically, the extracted probability would be based on the so-called Bayesian odds that a signal is present in the data, which requires the computationally costly evaluation of high-dimensional integrals [11]. By combining a sufficiently large number of these probability measurements, one can determine the rate of black hole mergers-a piece of information that can't be recovered from the traditional cross-correlation approach. Their method can also be used to produce more fine-grained information such as the rate of black hole mergers as a function of mass.

Smith and Thrane estimate that the improved search would give a reliable measure of the binary black hole background with just one day of data, while a cross-correlation search would take roughly four years. But this vast improvement in sensitivity comes at a huge increase in computational time because joint Bayesian analyses are so intensive. Nevertheless, the researchers calculated the computational cost and determined that it is within reach of LIGO and Virgo's computer resources. (The implementation of this new analysis method is under active consideration by the LIGO and Virgo scientific collaborations.) It may also be possible to generalize the new approach to cover signals that overlap in the detector, such as the much-longer-duration neutron star mergers.

Gold-plated detections such as the first one LIGO recorded in 2015 are what garner headlines and win Nobel Prizes. But Smith and Thrane have shown that clever data mining techniques can yield pay dirt from much more "modest" signals.

This research is published in Physical Review X.

\section{REFERENCES}

[1] B. P. Abbott et al. (LIGO Scientific Collaboration and Virgo Collaboration), "Observation of Gravitational Waves from a Binary Black Hole Merger," Phys. Rev. Lett. 116, 061102 (2016); B.P. Abbott et al. (LIGO Scientific Collaboration and Virgo Collaboration), "Observation of Gravitational Waves from a 22Solar-Mass Binary Black Hole Coalescence," Phys. Rev. Lett. 116, 241103 (2016).

[2] B. P. Abbott et al. (LIGO Scientific Collaboration and Virgo Collaboration), "Binary Black Hole Mergers in the First Advanced LIGO Observing Run," Phys. Rev. X 6, 041015 (2016).

[3] B.P. Abbott et al. (LIGO Scientific Collaboration and Virgo Collaboration), "GW170104: Observation of a 50-Solar-Mass Binary Black Hole Coalescence at Redshift 0.2," Phys. Rev. Lett. 118, 221101 (2017).

[4] B. P. Abbott et al. (LIGO Scientific Collaboration and Virgo Collaboration), "GW170608: Observation of a 19 Solar-mass Binary Black Hole Coalescence," Ap. J. Lett. 851, L35 (2017).

[5] B. P. Abbott et al. (LIGO Scientific Collaboration and Virgo Collaboration), "GW170814: A Three-Detector Observation of Gravitational Waves from a Binary Black Hole Coalescence," Phys. Rev. Lett. 119, 141101 (2017).

[6] B. P. Abbott et al. (LIGO Scientific Collaboration and Virgo Collaboration), "GW170817: Observation of Gravitational Waves from a Binary Neutron Star Inspiral," Phys. Rev. Lett. 119, 161101 (2017).

[7] R. Smith and E. Thrane, "Optimal Search for an Astrophysical Gravitational-Wave Background," Phys. Rev. X 8, 021019 (2018).

[8] C. Messenger and J. Veitch, "Avoiding Selection Bias in Gravitational Wave Astronomy," New J. Phys. 15, 053027 (2013).

[9] W. M. Farr, J. R. Gair, I. Mandel, and C. Cutler, "Counting and Confusion: Bayesian Rate Estimation with Multiple Populations," Phys. Rev. D 91, 023005 (2015).

[10] B.P. Abbott et al. (LIGO Scientific Collaboration and Virgo Collaboration), "GW150914: Implications for the Stochastic Gravitational-Wave Background from Binary Black Holes," Phys. Rev. Lett. 116, 131102 (2016).

[11] T. B. Littenberg and N. J. Cornish, "Bayesian Approach to the Detection Problem in Gravitational Wave Astronomy," Phys. Rev. D 80, 063007 (2009).

10.1103/Physics. 11.36 